LANC-TH9512 astro-ph/9501113

\title{
THE GRISHCHUK-ZELDOVICH EFFECT IN THE OPEN UNIVERSE
}

\author{
David H. Lyth \\ School of Physics and Chemistry, \\ University of Lancaster, Lancaster LA1 4YB. U. K.
}

\section{Introduction}

When considering perturbations in an open universe, cosmologists retain only subcurvature modes (defined as eigenfunctions of the Laplacian whose eigenvalue is less than -1 in units of the curvature scale, in contrast with the super-curvature modes whose eigenvalue is between -1 and 0 ). Mathematicians have known for almost half a century that all modes must be included to generate the most general homogeneous Gaussian random field, despite the fact that any square integrable function can be generated using only the sub-curvature modes. The former mathematical object, not the latter, is the relevant one for physical applications. This article summarizes recent work with A. Woszczyna. The mathematics is briefly explained in a language accessible to physicists. Then the effect on the cmb of any super-curvature contribution is considered, which generalizes to $\Omega_{0}<1$ the analysis given by Grishchuk and Zeldovich in 1978.

\section{The mode expansion}

According to the Einstein field equation, the energy density $\Omega$ of the universe is given by

$$
1-\Omega=-\frac{K}{(a H)^{2}}
$$

Here $K$ is a constant, $H=\dot{a} / a$ is the Hubble parameter, and $\Omega$ is the energy density measured in units of the critical density $3 H^{2} / 8 \pi G$. The spatial curvature scalar is $R^{(3)}=6 K / a^{2}$ and we set $K=-1$ so that $a$ is the curvature scale. Then the case $\Omega=1$ corresponds to the limit $a \rightarrow \infty$, with physical distances like $H^{-1}$ remaining constant.

We are interested in the stochastic properties of the perturbations, at fixed time. To define them we will take the approach of considering an ensemble of universes of which ours is supposed to be one. If, in some region of space, a perturbation $f$ can be written as a sum of terms, with the coefficient of each term having an independent Gaussian probability distribution, it is a Gaussian random field, and its stochastic properties are completely determined by its correlation function. There 
is no requirement that the region of space be infinite, or that the terms be linearly independent. If the correlation function depends only on the geodesic distance between the points, the field is said to be homogeneous with respect to the group of transformations that preserve this distance. We assume that each cosmological perturbation in the observable universe is a typical realization of some homogeneous Gaussian random field.

Spherical coordinates are defined by the line element

$$
\mathrm{d} l^{2}=a^{2}\left[\mathrm{~d} r^{2}+\sinh ^{2} r\left(\mathrm{~d} \theta^{2}+\sin ^{2} \theta \mathrm{d} \phi^{2}\right)\right]
$$

Any homogeneous Gaussian random field can be generated ${ }^{1,2,3}$ by expanding it in eigenfunctions of the Laplacian with eigenvalue $(k / a)^{2}<0$,

$$
f(r, \theta, \phi, t)=\int_{0}^{\infty} \mathrm{d} k \sum_{l m} \tilde{f}_{k l m}(t) Z_{k l m}(r, \theta, \phi)
$$

Here $q^{2}=k^{2}-1$, and the mode functions are $Z_{k l m}=\Pi_{k l}(r) Y_{l m}(\theta, \phi)$. For $q^{2}>0$ the radial functions are

$$
\begin{aligned}
\Pi_{k l} & \equiv N_{k l} \tilde{\Pi}_{k l} \\
\tilde{\Pi}_{k l} & \equiv|q|^{-2}(\sinh r)^{l}\left(\frac{-1}{\sinh r} \frac{\mathrm{d}}{\mathrm{d} r}\right)^{l+1} \cos (q r) \\
N_{k l} & \equiv \sqrt{\frac{2}{\pi}}|q|\left[\prod_{n=1}^{l}\left(q^{2}+n^{2}\right)\right]^{-1 / 2} \quad(l>0)
\end{aligned}
$$

with $N_{k 0} \equiv \sqrt{2 / \pi}|q|$. For $-1<q^{2}<0, \cos (q r)$ is replaced by $\cosh (|q| r)$. The spectrum $\mathcal{P}_{f}$ is defined by

$$
\left\langle\tilde{f}_{k l m}^{*} \tilde{f}_{k^{\prime} l^{\prime} m^{\prime}}\right\rangle=\frac{2 \pi^{2}}{k\left|q^{2}\right|} \mathcal{P}_{f}(k) \delta\left(k-k^{\prime}\right) \delta_{l l^{\prime}} \delta_{m m^{\prime}}
$$

and the correlation function is

$$
\xi_{f}(r)=\int_{0}^{\infty} \frac{\mathrm{d} k}{k} \mathcal{P}_{f}(k) \frac{\sin (q r)}{q \sinh r}
$$

For $r \gg 1$, the contribution to the correlation function from a mode with $k^{2} \ll 1$ is $\xi_{f}(r) \propto \exp \left(-k^{2} r\right)$ Thus the correlation length, in units of the curvature scale $a$, is of order $k^{-2}$. This is in contrast with the flat-space case, where the contribution from a mode with $k \ll 1$ gives a correlation length of order $1 / k$.

\section{The Grishchuk-Zeldovich effect}

The cmb anisotropy is generally ascribed to a primeval curvature perturbation (measured by comoving observers), conveniently taken to be $\mathcal{R}$ defined by

$$
4\left(k^{2}+3\right) \mathcal{R}_{k l m} / a^{2}=\delta R_{k l m}^{(3)}
$$


In the limit $\Omega \rightarrow 1$,

$$
4 k^{2} \mathcal{R}_{k l m} / a^{2}=\delta R_{k l m}^{(3)}
$$

During matter domination $\mathcal{R}$ is constant until $\Omega$ breaks away from 1 . For $l \lesssim 30$ the mean square multipole $C_{l}$ of the cmb anisotropy is given by the Sachs-Wolfe approximation

$$
\begin{aligned}
C_{l} & =2 \pi^{2} \int_{0}^{\infty} \frac{\mathrm{d} k}{k} \mathcal{P}_{\mathcal{R}}(k) I_{k l}^{2} \\
|q| I_{k l} & =\frac{1}{5} \Pi_{k l}\left(\eta_{0}\right)+\frac{6}{5} \int_{0}^{\eta_{0}} \mathrm{~d} r \Pi_{k l}(r) F^{\prime}\left(\eta_{0}-r\right) \\
F(\eta) & =5 \frac{\sinh ^{2} \eta-3 \eta \sinh \eta+4 \cosh \eta-4}{(\cosh \eta-1)^{3}}
\end{aligned}
$$

with $\eta=2(a H)^{-1}$ and $r=\eta_{0}$ the last scattering surface. In this regime the COBE measurements give $l^{2} C_{l} \simeq 8 \times 10^{-10}$. Within the observational uncertainties this is consistent $^{4}$ with a flat spectrum for all $0.1 \leq \Omega_{0} \leq 1.0$, of magnitude $\mathcal{P}_{\mathcal{R}} \sim 10^{-9}$ to $10^{-10}$. The corresponding mean square curvature perturbation $\left\langle\mathcal{R}^{2}\right\rangle$ is of the same order.

Now suppose that the spectrum $\mathcal{P}_{\mathcal{R}}$ rises sharply on some very large scale $k_{\mathrm{VL}}$ but that the perturbation is still a typical realization of a Gaussian random field so that it can be discussed using the above formalism. What is the effect on the cmb anisotropy? For $\Omega_{0}=1$ this question was asked and essentially answered by Grishchuk and Zeldovich ${ }^{5}$. The large scale contribution can be taken to be

$$
\mathcal{P}_{\mathcal{R}}^{\mathrm{VL}} \simeq \delta\left(\ln k-\ln k_{\mathrm{VL}}\right)\left\langle\mathcal{R}^{2}\right\rangle
$$

In a sphere whose radius is of order the correlation length $d_{\mathrm{VL}}=a_{0} / k_{\mathrm{VL}}, \mathcal{R}$ is roughly constant with typical value $\left\langle\mathcal{R}^{2}\right\rangle^{1 / 2}$. From Eq. (10) it is of order $d_{\mathrm{VL}}^{2} \delta R^{(3)}$, which is a dimensionless measure of the geometry distortion due to the perturbation (recall that the background curvature $R^{(3)}$ vanishes for $\Omega_{0}=1$ ). For instance it is roughly equal to the fractional departure from $4 \pi d_{\mathrm{VL}}^{2}$ of the sphere's area. The maximal distortion, corresponding to regions of positive curvature closing on themselves, is $\left\langle\mathcal{R}^{2}\right\rangle \lesssim 1$. The quadrupole dominates for $d_{\mathrm{VL}} \gg H_{0}^{-1}$, and is given by

$$
C_{2}^{\mathrm{VL}} \sim\left(d_{\mathrm{VL}} H_{0}\right)^{-4}\left\langle\mathcal{R}^{2}\right\rangle
$$

If the geometry distortion is maximal, then $d_{\mathrm{VL}} H_{0} \sim\left(C_{2}^{\mathrm{VL}}\right)^{1 / 4} \gtrsim 10^{2}$. In words, the correlation length is then more than two orders of magnitude bigger than the size of the observable universe.

To generalize this analysis to $\Omega_{0}<1$ one needs to take the background spatial curvature into account, and to note that the limit of large scales corresponds to $k \rightarrow 0$, not $q \rightarrow 0$. This has not been done to date. The only relevant publications of which we are aware either ignore the spatial curvature ${ }^{6}$ or take use the $q \rightarrow$ 0 limit $^{7,8}$. Consider therefore Eq. (14) with $k_{\mathrm{VL}} \ll 1$, and suppose that $\Omega_{0}$ is significantly below 1 so that $a_{0} H_{0} \sim 1$. In the absence of perturbations the geometry distortion of the observable universe is $a_{0}^{2} R^{(3)} \sim 1$, and the addition distortion caused by the perturbation is $\sim a_{0}^{2} \delta R^{(3)} \sim \mathcal{R}$. Thus, $\mathcal{R}$ measures the fractional 
change in the geometry distortion, and since the relation between $\delta R^{(3)}$ and $\mathcal{R}$ is now scale independent this remains true on larger scales. The maximal distortion, corresponding to regions of space closing on themselves, is still $\left\langle\mathcal{R}^{2}\right\rangle \lesssim 1$.

As $k \rightarrow 0, \Pi_{k 0} \rightarrow 1$, but the other radial functions are proportional to $k$. Defining $I_{l} \equiv \lim _{k \rightarrow 0} I_{k l} / k$,

$$
C_{l}^{\mathrm{VL}}=I_{l}^{2} k_{\mathrm{VL}}^{2}\left\langle\mathcal{R}^{2}\right\rangle
$$

Since $I_{l}$ is roughly of order 1 for low multipoles, and also $a_{0} \sim H_{0}^{-1} \sim 1$ we can write this

$$
C_{l}^{\mathrm{VL}} \sim\left(d_{\mathrm{VL}} H_{0}\right)\left\langle\mathcal{R}^{2}\right\rangle
$$

The prefactor is not now raised to the fourth power as it is for $\Omega_{0}=1$, so that for maximal distortion $d_{\mathrm{VL}}$ must now be ten orders of magnitude bigger than the size of the observable universe! There are two physical reasons for the difference. One is that the correlation length is $a_{0} k_{\mathrm{VL}}^{-2}$ instead of $a_{0} k_{\mathrm{VL}}^{-1}$. The other is that the presence of background curvature allows the geometry distortion to be of order 1 in the observable universe, whereas before it was at most of order $k_{\mathrm{VL}}^{2} \ll 1$.

In the case $\Omega_{0}=1$, the Grishchuk-Zeldovich effect contributes only to the quadrupole, and is not seen in the data (ie., the quadrupole is not anomalously high). In the case $\Omega_{0}<1$ it contributes to all multipoles up to some maximum, which is probably within the regime of validity of the Sachs-Wolfe approximation. It would be worth evaluating the $l$ dependence to see whether it is the same as the observed $C_{l} \propto l^{-2}$ for some range of $\Omega_{0}$. If so the effect might be present, and one could see whether this was so by looking at higher multipoles. (A more bizarre possibility would be that the effect persists even beyond the range of the Sachs-Wolfe approximation, in which case a full calculation would be necessary. The formalism is already in place, and has already been used for the sub-curvature modes ${ }^{9,10,11}$.)

For ease of visualization we have used the concept of the correlation length $d_{\mathrm{VL}}$, which presupposes that the perturbation continues to be a typical realization of a Gaussian random field in a region around us whose size is bigger than $d_{\mathrm{VL}}$, and therefore much bigger than the observable universe. The effect is really calculated on the hypothesis that the perturbation is a typical realization of a Gaussian random field within the observable universe, and can be written in terms of $k_{\mathrm{VL}}$ without reference to a correlation length. However, if the hypothesis is valid for $k$ down to some minimum value, it is reasonable to suppose that it can indeed be extended out to a region bigger than the corresponding correlation length. Thus a positive detection of the Grishchuk-Zeldovich effect would suggest that this is the case. On the other hand a failure to detect the effect, which seems more likely, will tell us essentially nothing!

Finally, let us ask whether one should expect the effect to be present even below the level of detectability. For the case $\Omega_{0}=1$ the usual hypothesis is that the curvature perturbation comes from a vacuum fluctuation of the inflaton field, and in 1990 this was extended ${ }^{13}$ to the case $\Omega_{0}<1$. To the extent that this is true there are no super-curvature modes, which means that for $\Omega_{0}$ appreciably less than 1 there is no Grishchuk-Zeldovich effect. Like any hypothesis in physics this will be at best an approximation, and it will fail above some large scale. (As we just discussed, 'scale' strictly means simply some large value of $k^{-1}$, but one can probably think 
of it a also a large correlation length.) However, the hypothesis that the curvature perturbation in the observable universe is a typical realization of a homogeneous Gaussian random field will also fail above some large scale, and this might well be the same as the scale on which the vacuum fluctuation hypothesis fails. If so, there will be no Grishchuk-Zeldovich effect.

It would be instructive to see how all this works in bubble model ${ }^{13}$ of the $\Omega<1$ universe. According to this model we inhabit the interior of the bubble extending far beyond the observable universe. Within the bubble the perturbation is well approximated by a typical realization of a random Gaussian field, which has only sub-curvature modes because it originates as a vacuum fluctuation. As the boundary is approached the nature of the perturbation changes and it no longer corresponds to a typical realization of the random field. Thus one expects in this model the coincidence of scales mentioned in the last paragraph, and no Grishchuk-Zeldovich effect.

\section{ACKNOWLEDGEMENTS}

This work was started with the help of EU research grant ERB3519PL920782(10835). One of us (DHL) thanks the Isaac Newton Institute for a visiting Fellowship while the work was being completed, and Bruce Allen, Robert Caldwell, Misao Sasaki and Neil Turok for useful discussions.

\section{REFERENCES}

1. YAGLOM, M. 1961 in Proceedings of the Fourth Berkeley Symposium Volume II, J. Neyman, Ed. University of California Press, Berkeley.

2. KREIN, M. G. 1949. Ukrain. Mat. Z. 1, No. 1, 64; ibid 1950 2, No. 1, 10.

3. LYTH, D. H. \& A. WOSZCZYNA. preprint astro-ph/9501044, submitted to Phys. Rev. D.

4. KAMIONKOWSKI, M., D. N. SPERGEL \& N. SUGIYAMA. 1994. Astrophys. J. 426, L57; GORSKI, K. M., H. RATRA, N. SUGIYAMA \& A. J. BANDAY, preprint.

5. GRISHCHUK, L. P. \& Ya. B. ZELDOVICH. 1978. Astron. Zh. 55, 209 [Sov. Astron. 22, 125 (1978)].

6. TURNER, M. S.. 1991. Phys Rev D 44, 12.

7. KAMIONKOWSKY, M. \& D. N SPERGEL. 1994. Astrophys. J. 432, 7.

8. KASHLINSKY, A., I. TKACHEV \& J. FRIEDMAN. 1994. Phys. Rev. Lett., 73, 1582.

9. SUGIYAMA, N. \& J. Silk. 1994. Phys. Rev. Lett. 73, 509.

10. KAMIONKOWSKI, M., D. N. SPERGEL \& N. SUGIYAMA. 1994. Astrophys. J. 426, L57.

11. GORSKI, K. M. , H. RATRA, N. SUGIYAMA \& A. J. BANDAY, preprint.

12. LYTH, D. H.\& E. D. STEWART. 1990. Phys Lett. B 252, 336.

13. COlEMAN, S. \& F. DE LUCCIA. 1980. Phys. Rev. D 21, 3305; GOTT, J. R.. 1982. Nature 295 , 304; GUTH, A. H. \& E. J. WEINBERG. 1983. Nucl. Phys. B212, 321; GOTT, J. R. \& T. S. STATLER. 1984. Phys. Lett. B136, 157; SASAKI, M., T. TANAKA, K. YAMAMOTO \& J. YOKOYAMA. 1993. Phys. Lett. B 317, 510; SASAKI, M., T. TANAKA, K. YAMAMOTO \& J. YOKOYAMA. 1993. Prog. Theor. Phys. 90, 1019; BUCHER, M., A. GOLDHABER \& N. TUROK. 1994. preprint; TANAKA, T. \& M. SASAKI. 1994. two preprints; YAMAMOTO, K., T. TANAKA, \& M. SASAKI. 1994. preprint. 